Background Neonatal abstinence syndrome (NAS) is a complex of symptoms in newborns exposed to substances/drugs in-utero or after birth. Clonidine is a central alpha- 2 agonist and recent studies have shown it can decrease NAS symptoms in opiate withdrawal.

Objective To determine the efficacy of clonidine as an adjunctive agent to phenobarbitale (PB). To elucidate demographic factors, maternal drug profile, nature of the symptoms in infants. To compare NAS profile with $\mathrm{PB}$ and $\mathrm{PB}+$ clonidine. To show associated side effects with clonidine.

Design/Methods Retrospective review of infants $\geq 35$ weeks GA admitted to HSC, Winnipeg from January 2005 to July 2010. Abstinence scores 20 hours before and 40 hours after $\mathrm{PB}$ and $\mathrm{PB}+$ clonidine were measured by Finnegan scoring system and compared by ANOVA.

Results Twenty four infants (GA 39.3 \pm 1.4 wks, BW 3316 $\pm 595 \mathrm{~g}$ ) were treated by $\mathrm{PB}+$ clonidine combination. Fifty eight percent exposed to multiple drugs. Methadone was the most common drug of exposure. Tremor, increased tone, regurgitation and poor feeding were common symptoms. When $\mathrm{PB}$ was used alone as initial therapy, NAS scores increased from $6.9 \pm 3.3$ to $7.5 \pm 3.0$ ( $p>0.05$ ) at pre and post medication periods respectively. Clonidine was added to $\mathrm{PB}$ at 3.5 to $5.3 \mathrm{mg} / \mathrm{kg} / \mathrm{day}$ and NAS scores were decreased from $8.7 \pm 3.4$ to $7 \pm 3.5(p<0.001)$. There were no recorded side effects for clonidine.

Conclusions Our study suggests that clonidine may be a useful adjunctive treatment of NAS in infants who respond incompletely to PB. Cardiovascular side effects were not common in our study.

\section{UNLICENSED AND OFF-LABEL MEDICATION USE IN THE NEONATAL INTENSIVE CARE UNIT: A PROSPECTIVE COHORT STUDY}

doi:10.1136/archdischild-2012-302724.1647

1,2,3E Kieran, ' $\mathrm{N}$ O' Callaghan, ${ }^{1,2,3} \mathrm{CP} \mathrm{O}^{\prime}$ Donnell. 'Neonatology, The National Maternity Hospital; ${ }^{2}$ The National Children's Research Centre, Crumlin; ${ }^{3}$ School of Medicine and Medical Sciences, University College Dublin; ${ }^{2}$ hharmacy, The National Maternity Hospital, Dublin, Ireland

Background Many medications have not been extensively studied in children. Medications may be prescribed for indications for which their use has not been approved (unlicensed use); or may be given for an approved indication, but to an age group or at a dose or frequency or by a route that has not been approved (off-label use). We wished to determine the extent of unlicensed and off-label prescription in our NICU.

Methods We prospectively studied infants admitted to our tertiary level NICU over 2 months. We recorded demographic data and all medications prescribed for all infants. We compared the use of each medication to their licensed indications as contained in the Summary of Product Characteristics.

Results 110 infants were admitted. All infants received a prescribed medication. 79 different medications were prescribed to these 110 infants, a median (IOR) of $4(3,12)$ each. $41 \%$ of prescribed medications were given in an unlicensed manner and $15 \%$ in an off-label manner. 12 (11\%) infants received an unlicensed medication, $5(4 \%)$ infants received an off-label medication and 38 (35\%) infants received both an unlicensed and off-label medication. Of infants $<32$ weeks, $91 \%$ received unlicensed and $85 \%$ off-label medications. $100 \%$ of infants $<28$ weeks received an unlicensed and an off-label medication. There were 2 adverse events related to medications.

Conclusions Most medications prescribed to newborn are unlicensed or given for off-label indications. Many infants, and the majority of preterm infants, admitted to NICU receive unlicensed and off-label prescriptions. Adverse events appear uncommon.

\section{CLINICAL COURSE AND DRUG SUSCEPTIBILITY FOR INFANTS WITH UREPALSMA INFECTION}

doi:10.1136/archdischild-2012-302724.1648

SM Lee, HS Eun, R Namgung, MS Park, KI Park, C Lee. Pediatrics, Yonsei University College of Medicine, Seoul, Republic of Korea

Objectives Ureaplasma species were associated bronchopulmonary dysplasia in preterm infants. We aim to analyze the antibiotic susceptibility of ureaplasma urealyticum and clinical manifestations in preterm infants with ureaplasma urealyticum colonization.

Methods In a retrospective study, 416 preterm infants ( $\leq$ gestation $32 \mathrm{wk}$ ) and their mothers admitted to Severance Children's Hospital and Gangnam Severance Hospital NICU between Jan 2008. to Jun 2011, were reviewed. Ureaplasma test was done by culture for mothers and PCR in urine and tracheal aspirates for preterm infants. Ureaplasma colonization was confirmed $7.5 \%$ of infants, and $31 \%$ of the mothers. If positive result was noted, all infants were initially treated with erythromycin empirically.

Results Thirty one infants who had positive ureaplasma PCR test $(28.3 \pm 3.1 \mathrm{wk}, 1050 \pm 490 \mathrm{~g})$ and 385 infants with negative test $(29.0 \pm 3.2 \mathrm{wk}, 1190 \pm 550 \mathrm{~g})$ were recruited as controls. Infants with ureaplasma infection had longer durations of oxygen administration $(p=0.039)$ and mechanical ventilation $(p=0.041)$. The incidence of pathologic chorioamnionitis were significantly higher $(p<0.001)$. Infants with ureaplasma infection had higher incidence of moderate/severe BPD. For antimicrobial susceptibility, $23 \%$ of erythromycin resistance, $16 \%$ of zithromycin resistance, $38 \%$ of ciprofloxacin resistance and no jasamycin resistance were shown. Among 31 infants with erythromycin treatment, 18 (58\%) of susceptible, $6(19 \%)$ of intermediate were cured after 13 days of treatment, and 4 showed poor response erythromycin treatment, 2 changed to josamycin and 2 infants to clarithromycin and all were completely treated.

Conclusion Ureaplasma colonized infants showed higher incidence of BPD. Proper antimicrobial use may reduce the morbidity associated with ureaplasma colonization.

\section{RETROSPECTIVE ANALYSIS OF DOXAPRAM FOR THE TREATMENT OF APNEA OF PREMATURITY}

doi:10.1136/archdischild-2012-302724.1649

1J Winter, 'B Fekker, ${ }^{2} \mathrm{~A}$ Gerhold-Ay, 'A Kidszun, ${ }^{2}$ Schmidtmann, 'C Whybra-Trümpler, ${ }^{1}$ E Mildenberger. 'Neonatology, Children's Hospital; '2Institute of Medical Biometry, Epidemiology and Informatics, University Medical Center of the Johannes Gutenberg University, Mainz, Germany

Background and Aims Only one small randomized controlled study on doxapram to treat apnea of prematurity is available. Before the implementation of a local treatment protocol, we aimed to evaluate the frequency of administration of doxapram in our NICU. We asked, if frequency and severity of apneas were affected by doxapram, if intubation for apnea was avoided, and if side effects occurred.

Methods We retrospectively analysed all premature infants $<30$ weeks treated with doxapram during 03/2008 to 03/2010. We registered the number of apneas, bradycardias, and desaturations, an hour before, at the start of, and during 48 hours after onset of treatment.

Results 17 of 64 (27\%) infants (mean gestational age 26.1 weeks, mean birth weight 733g) were treated during two years. All of them had been treated with caffeine before doxapram was applied. 70 therapy courses of 16 infants were analyzed. In 8 of 70 (11\%) therapy courses, infants were intubated because of apnea during 48 hours of doxapram treatment. The frequency of apneas ( $2.24 \mathrm{vs}$. 0.17 ), bradycardias $<80 / \mathrm{min}(0.93$ vs. 0.14$)$, and desaturations 